\title{
Semeadora-adubadora: Mecanismos de corte de palha e cargas verticais aplicadas
}

\author{
Paulo R. A. Silva ${ }^{1}$, Sérgio H. Benez ${ }^{1}$, Samir P. Jasper ${ }^{2}$, \\ André S. Seki ${ }^{1}$, Fabrício C. Masiero ${ }^{1} \&$ Neilor B. Riquetti ${ }^{1}$
}

RESU M O

Realizou-se este trabalho com o objetivo de avaliar o desempenho dos mecanismos de corte de palha de uma semeadora-adubadora em diferentes coberturas vegetais e cargas verticais aplicadas. 0 delineamento experimental utilizado foi em blocos ao acaso e esquema fatorial $3 \times 4$, com 4 repetições. 0 s trabalhos foram realizados com três diferentes mecanismos de corte de palha (disco liso, disco corrugado e disco ondulado) utilizando-se quatro diferentes cargas verticais (750, 1500, 2250 e $3000 \mathrm{~N}$ ) em cinco coberturas de palha (milho, sorgo, aveia preta, triticale e nabiça). Os resultados mostraram que quanto maior a quantidade de matéria seca da cobertura vegetal maior também é a demanda de forças horizontal e vertical. 0 disco de corte ondulado possibilita os maiores valores de área de solo mobilizado, exige maiores valores de força horizontal, força vertical e força horizontal por profundidade de corte dos discos (força específica). 0 tratamento com disco liso exigiu maiores valores de força horizontal por área de solo mobilizado e de força vertical nas coberturas de milho e sorgo.

Palavras-chave: plantio direto, cultura de cobertura, interação ferramenta-solo

\section{Seedrill: Mechanism of culting straw and applied vertical loads}

\begin{abstract}
A B ST RACT
This work had as objective to evaluate the performance of mechanisms of cutting of the no-tillage seedrills in different vegetable coverings and applied vertical loads. The experimental design used was in randomized blocks and factorial scheme $3 \times 4$, with four replications. The trials were accomplished with three different mechanisms of culting of the straw (plane disc, corrugated disc and wavy disc), using four different vertical loads $(750,1500,2250$ and $3000 \mathrm{~N})$. All treatments were accomplished in five straw coverings (corn, sorghum, triticale, black oats and radish). The results showed that larger the amount of dry matter covering the soil, higher the demand of horizontal and vertical forces. The wavy disc promotes the largest values of area of mobilized soil and requires large horizontal force, vertical force and horizontal force per area of cutting depth of discs (specific force). The treatment with plane disc required higher values of the horizontal forces per area of mobilized soil and of vertical force in the corn and sorghum coverings.
\end{abstract}

Key words: no-tillage, cover crop, tool-soil interaction 


\section{INTRODUÇÃO}

A maior incidência de utilização da mecanização agrícola ocorre no preparo do solo, que tem o objetivo de otimizar suas condições para germinação das sementes e a instalação das culturas. Além disso, a escolha do método de preparo do solo e do equipamento a ser utilizado pode auxiliar no controle das perdas de água e solo (Coelho et al., 2012; Llanillo et al., 2006; Gabriel Filho et al., 2010).

No sistema de plantio direto o tráfego de maquinário agrícola é reduzido em relação ao sistema de preparo convencional mas também pode influenciar as propriedades dos solos, ainda que apenas as operações de semeadura e colheita sejam realizadas já que os rodados dos tratores e colheitadeiras têm grande potencial de compactação do solo em superfície e subsuperfície (Schuler \& Wood, 1992; Santos et al., 2008). Solos mais compactados aumentam o desempenho de tração dos pneus porém requerem mais esforço para serem mobilizados, aumentam o desgaste de máquinas e equipamentos e podem prejudicar ou mesmo impedir o desenvolvimento radicular das plantas (Silva et al., 2000) e torná-las, assim, mais suscetíveis a perdas de produtividade em condições de deficiência hídrica (Beutler et al., 2005).

No caso de semeadoras-adubadoras o maior esforço de tração pode resultar, também, do aumento na profundidade de atuação dos órgãos ativos, especialmente discos de corte de resíduo e sulcadores para abertura de sulcos de adubação e semeadura (ASAE, 1999). Mello et al. (2003) verificaram que o mecanismo de haste sulcadora apresentou maior capacidade de mobilizar o solo e provocou reduções na sua densidade e resistência à penetração, além de aumento da macroporosidade. De acordo com esses autores o uso da haste sulcadora promove acréscimo de $11,3 \%$ na produtividade do milho em relação à obtida com uso de sulcador de discos. O uso de disco de corte de palha e de sulcadores do tipo facão, apesar de permitir operar em superfície com cerca de $8000 \mathrm{~kg} \mathrm{ha}^{-1} \mathrm{de}$ resíduos, resulta em exposição de cerca de $40 \%$ da superfície de solo em razão da mobilização do solo no sulco (Aratani et al., 2006).

A qualidade do corte da palha está relacionada às condições do solo, da palha e da semeadora. Para o corte adequado o solo deve ser um anteparo à ação do disco de corte e as coberturas do solo devem estar verdes ou secas, já que aquelas que se encontram murchas apresentam maior resistência ao corte (Siqueira \& Casão Júnior, 2004). Portanto, há necessidade do desenvolvimento de semeadoras adequadas às condições de trabalho, desde que capazes de cortar a palha, romper o solo, depositar as sementes e o adubo, fechar o sulco e compactar o solo sobre a semente.

A importância de ensaiar, dinâmica e individualmente, ferramentas constituintes de semeadora-adubadora em condições de campo, motivou a realização deste trabalho cujo objetivo foi avaliar o desempenho de discos de corte de palha de semeadora-adubadora em diferentes coberturas vegetais e cargas verticais aplicadas em um solo de textura argilosa.

\section{Material e MÉTODOS}

$\mathrm{O}$ experimento foi conduzido em área pertencente à Faculdade de Ciências Agronômicas da UNESP, na Fazenda Experimental Lageado (Botucatu, SP) durante o ano agrícola de 2005-2006. A área se localiza geograficamente entre as coordenadas $22^{\circ} 41^{\prime}$ Latitude Sul e 48 $34^{\circ}$ 'de Longitude Oeste, altitude média de $770 \mathrm{~m}$ e o clima classificado, segundo Koopler, como Cwb. Antecedendo ao ensaio foram semeadas em junho 2005, para a formação de palha na área experimental, as culturas do triticale (Triticum triticosecale), aveia preta (Avena strigosa Schreb) e nabiça (Raphanus raphanistrum); em setembro do mesmo ano a cultura do sorgo (Sorghum bicolor) e, em novembro de 2005, a cultura do milho (Zea mays L.). A coleta dos dados foi realizada sobre os restos vegetais dessas culturas após sua colheita. Os resultados da análise granulométrica na camada de 0,00-0,20 m apresentaram os valores médios de 106, 234 e $660 \mathrm{~g} \mathrm{~kg}^{-1}$ para areia, silte e argila, respectivamente, sendo o solo classificado como argiloso.

Utilizou-se, visando à obtenção dos dados de força de tração um chassi de subsolador disponível no mercado para a construção da barra porta-ferramentas a qual possui $1,240 \mathrm{~m}$ de comprimento, 0,985 $\mathrm{m}$ de largura e 1,285 m de altura, com peso total de aproximadamente $7400 \mathrm{~N}$, conforme Figura 1. Este equipamento foi acoplado ao sistema hidráulico do trator marca John Deere, modelo 6600, com potência na barra de tração de $98 \mathrm{~kW}$.

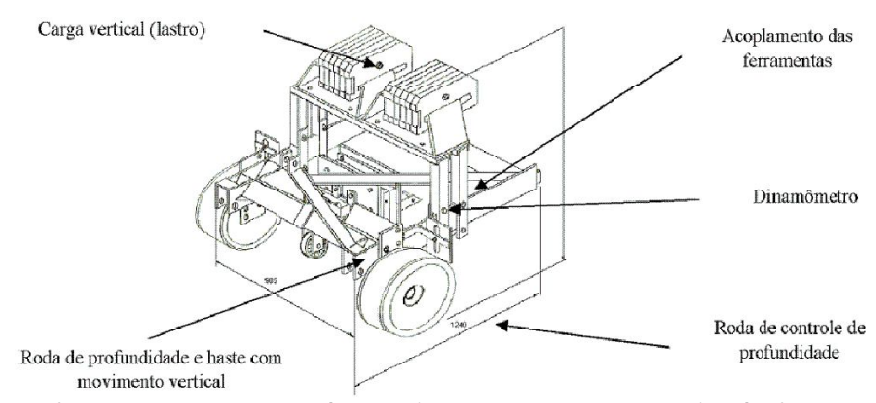

Figura 1. Esquema do equipamento para ensaio de ferramentas rompedoras de solo

Empregou-se o delineamento experimental fatorial em blocos ao acaso com dois fatores $(3 \times 4)$ sendo os tratamentos compostos da combinação dos três discos de corte liso, estriado e ondulado e de quatros níveis de cargas verticais $(750,1500,2250$ e $3000 \mathrm{~N})$.

Avaliou-se a profundidade de penetração dos discos utilizando-se um sensor constituído de um potenciômetro de giro fixado em uma chapa de aço contendo um eixo giratório no qual foi instalada uma roda em paralelo com a haste para monitoramento dos movimentos verticais, conforme a variação da superfície do solo (Figura 2).

Para mensurar as forças dos mecanismos de corte de palha foi utilizado um dinamômetro (Figura 3) equipado com extensômetros elétricos de resistência. Esses extensômetros foram ligados em pontes de Wheatstone, uma para cada força medida e momento, ou seja, Fx, Fy, Fz, My e Mz. 
Segundo Siqueira \& Casão Júnior (2004) os discos ondulados não apenas apresentam maior superfície de contato necessitando, com isto, de maior peso para penetração mas abrem sulcos mais largos ao cortar o material, mobilizando maior volume de solo.

Resultados semelhantes foram obtidos por Mion \& Benez (2008) que, estudando diferentes mecanismos de penetração no solo em diferentes cargas verticais aplicadas, concluíram que quanto maior a carga aplicada maior também é a área mobilizada de solo pelos sulcadores.

Embora não se tendo feito comparação entre as culturas estudadas, nota-se que os maiores valores de área de solo mobilizado ocorreram na cultura da nabiça cujos resultados podem ser explicados pela menor quantidade de massa seca da nabiça, obtendo maior penetração dos discos de corte no solo mobilizando, daí, maior área de solo.

\section{Profundidade de penetração dos discos}

Na Tabela 2 são apresentados os valores médios da variação da profundidade de penetração dos discos no solo nas diferentes cargas verticais aplicadas; observa-se que houve diferença significativa para todas as coberturas vegetais estudadas, embora se verifique, para todas as culturas, que quanto maior a carga vertical aplicada maior também a profundidade de penetração dos discos.

O disco ondulado obteve os menores valores de profundidade de penetração em relação aos demais, o disco liso obteve os maiores valores nas culturas do milho, aveia preta e nabiça e o disco de corte corrugado obteve os maiores valores para as coberturas do sorgo e do triticale, evento passível de ser explicado pelo fato do disco liso e do disco corrugado possuírem menor ângulo de corte resultando em maior eficiência do corte da palha.

Os maiores valores médios gerais de profundidade de penetração dos discos foram obtidos para a cobertura da nabiça seguida do triticale, aveia preta, milho e sorgo. Esses resultados podem ser explicados em razão da menor quantidade de massa seca da nabiça facilitando a penetração dos discos no solo.

Os menores valores obtidos na cobertura com sorgo se devem à dificuldade de corte da palha do sorgo em virtude de ser muito fibrosa e resistente. Apesar de possuir maior massa seca em relação às outras, a cobertura de aveia é de fácil corte e manejo.

Com base na não homogeneidade da quantidade de restos vegetais no solo, ocorrida na maioria das áreas de semeadura e pelos resultados obtidos neste experimento, constata-se que a semeadora deve ser regulada com pouco peso; para tal, a carga de adubo e semente deveria ser mínima para que não houvesse diferença nas profundidades de corte dos discos durante o processo de semeadura, o que não é realizado pela maioria dos produtores, o que se explica com base no fato de que, quando a semeadora corta a palha com o mínimo de carga, ela a cortará, também, quando estiver com a capacidade máxima limitando sua profundidade no eixo do disco de corte; caso contrário, quando regulada para o corte da palha na capacidade máxima, esta poderá não cortar a palha adequadamente se ocorrer diminuição do peso total, ou seja, com carga mínima, podendo ocorrer o embuchamento da máquina e o envelopamento das sementes.
Tabela 2. Valores médios ${ }^{*}$ de profundidade de penetração dos discos de corte $(\mathrm{m})$ para as diferentes coberturas vegetais

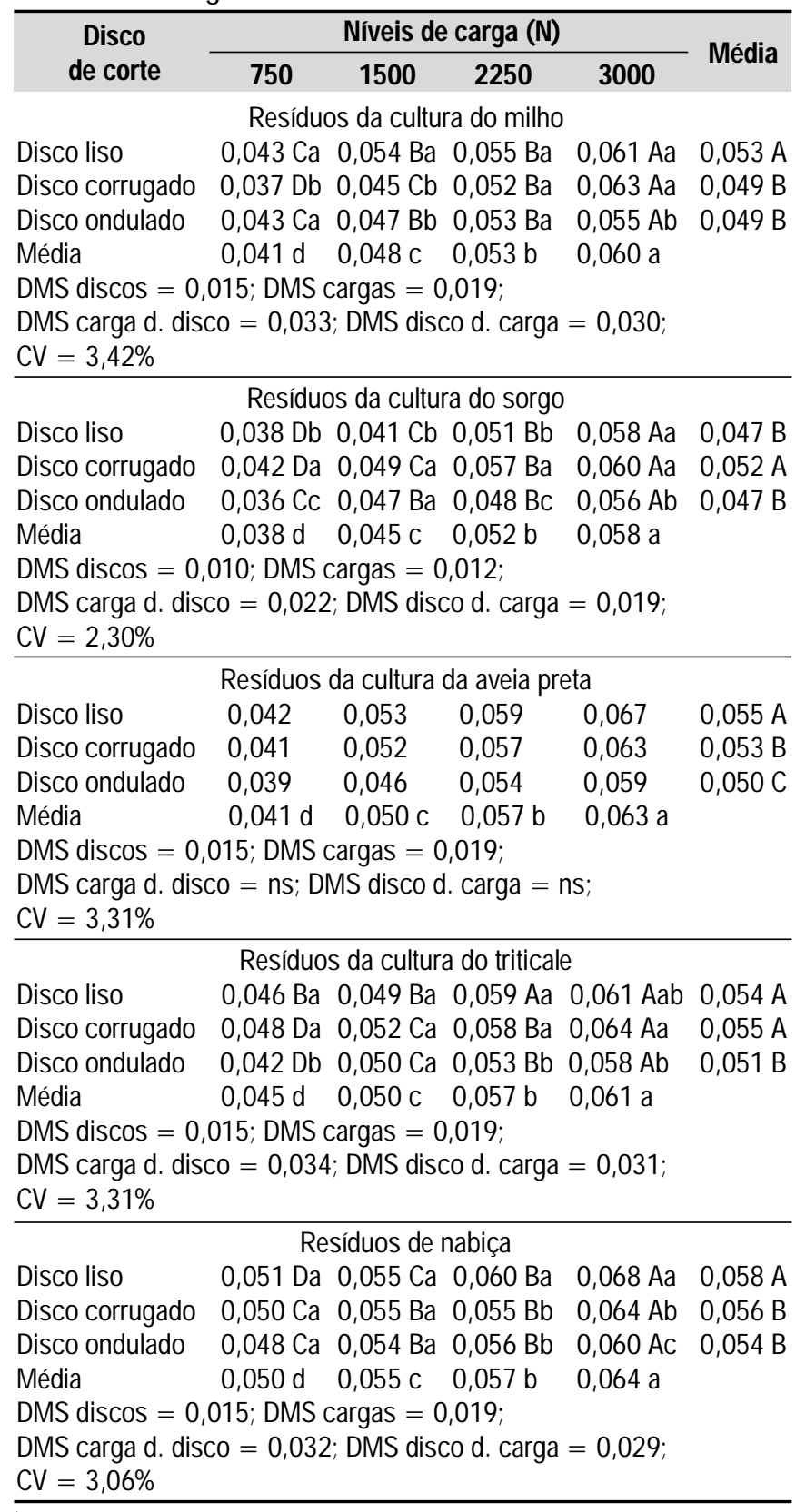

"Médias seguidas por letras distintas, maiúsculas nas linhas e minúsculas nas colunas, diferem pelo "Teste Tukey" a 0,05 de probabilidade

\section{Força horizontal}

Os resultados médios de força horizontal são apresentados na Tabela 3, através da qual se verifica que houve diferença estatística significativa para todas as coberturas vegetais estudadas.

Em relação aos discos de corte, os tratamentos com o disco ondulado obtiveram o maior requerimento de força horizontal para vencer a resistência do solo em comparação aos demais, possivelmente em virtude do desenho do disco e por sua ação dinâmica no solo serem diferentes das demais mobilizando, de certo modo, maior área de solo.

Os discos de corte do tipo liso e corrugado sinalizaram desempenho semelhante em relação aos valores de força 
Tabela 3. Val ores médios ${ }^{*}$ de força horizontal (N) para as diferentes coberturas vegetais

\begin{tabular}{|c|c|c|c|c|c|}
\hline \multirow{2}{*}{$\begin{array}{c}\text { Disco } \\
\text { de corte }\end{array}$} & \multicolumn{4}{|c|}{ Níveis de carga (N) } & \multirow{2}{*}{ Média } \\
\hline & 750 & 1500 & 2250 & 3000 & \\
\hline \multicolumn{6}{|c|}{ Resíduos da cultura do milho } \\
\hline Disco liso & $130 \mathrm{Bab}$ & $152 \mathrm{Ab}$ & $156 \mathrm{Ab}$ & $156 \mathrm{Ab}$ & $149 \mathrm{~B}$ \\
\hline Disco corrugado & $120 \mathrm{Cb}$ & $126 \mathrm{BCC}$ & $146 \mathrm{Ab}$ & $143 \mathrm{ABb}$ & $134 \mathrm{C}$ \\
\hline Disco ondulado & $138 \mathrm{Ca}$ & $181 \mathrm{Ba}$ & $197 \mathrm{Ba}$ & $222 \mathrm{Aa}$ & $185 \mathrm{~A}$ \\
\hline Média & $129 \mathrm{c}$ & $153 \mathrm{~b}$ & $166 a$ & 174 a & \\
\hline \multicolumn{6}{|c|}{$\begin{array}{l}\text { DMS discos = 8,33; DMS cargas = 10,59; } \\
\text { DMS carga d. disco = 18,36; DMS disco d. carga = 16,64; } \\
\text { CV }=16,15 \%\end{array}$} \\
\hline \multicolumn{6}{|c|}{ Resíduos da cultura do sorgo } \\
\hline isco liso & $140 \mathrm{Db}$ & $158 \mathrm{Bb}$ & $165 \mathrm{Cb}$ & $152 \mathrm{AC}$ & $154 \mathrm{~B}$ \\
\hline Igado & $137 \mathrm{Db}$ & $148 \mathrm{Cc}$ & $160 \mathrm{Bb}$ & $169 \mathrm{Ab}$ & D כJכו \\
\hline Disco ondulado & $151 \mathrm{Da}$ & $190 \mathrm{Ca}$ & $199 \mathrm{Ba}$ & $231 \mathrm{Aa}$ & \\
\hline Média & $142 \mathrm{~d}$ & $166 \mathrm{C}$ & $175 \mathrm{~b}$ & 184 a & \\
\hline \multicolumn{6}{|c|}{$\begin{array}{l}\text { DMS discos = 2,81; DMS cargas = 3,58; } \\
\text { DMS carga d. disco =6,19; DMS disco d. carga = 5,61; } \\
\text { CV }=19,38 \%\end{array}$} \\
\hline \multicolumn{6}{|c|}{ Resíduos da cultura da aveia preta } \\
\hline Disco liso & $121 \mathrm{Ba}$ & $143 \mathrm{Aa}$ & $146 \mathrm{Ab}$ & $147 \mathrm{Ab}$ & $139 \mathrm{~B}$ \\
\hline Disco corrugado & $99 \mathrm{Bb}$ & $104 \mathrm{Bb}$ & $143 \mathrm{Ab}$ & $141 \mathrm{Ab}$ & 122 \\
\hline Disco ondulado & $129 \mathrm{Ca}$ & $153 \mathrm{Ba}$ & $159 \mathrm{Ba}$ & $185 \mathrm{Aa}$ & $156 \mathrm{~A}$ \\
\hline Média & $117 d$ & $133 \mathrm{c}$ & $149 \mathrm{~b}$ & 158 a & \\
\hline \multicolumn{6}{|c|}{$\begin{array}{l}\text { DMS discos = 6,06; DMS cargas = } 7,71 ; \\
\text { DMS carga d. disco = 13,36; DMS disco d. carga = 12,12; } \\
C V=15,02 \%\end{array}$} \\
\hline \multicolumn{6}{|c|}{ Resíduos da cultura do triticale } \\
\hline Disco liso & $97 \mathrm{Ba}$ & $124 \mathrm{Aa}$ & $128 \mathrm{Ab}$ & $143 \mathrm{Ab}$ & $123 \mathrm{~B}$ \\
\hline Disco corrugado & $105 \mathrm{Ca}$ & $131 \mathrm{Ba}$ & $124 \mathrm{BCb}$ & $167 \mathrm{Aa}$ & $132 \mathrm{~B}$ \\
\hline Disco ondulado & $111 \mathrm{Ba}$ & $129 \mathrm{Ba}$ & $166 \mathrm{Aa}$ & $181 \mathrm{Aa}$ & $146 \mathrm{~A}$ \\
\hline Média & $104 \mathrm{C}$ & $128 \mathrm{~b}$ & $139 b$ & 164 a & \\
\hline \multicolumn{6}{|c|}{$\begin{array}{l}\text { DMS discos = 9,34; DMS cargas = 11,89; } \\
\text { DMS carga d. disco = 20,60; DMS disco d. carga = 18,68; } \\
\text { CV }=18,04 \%\end{array}$} \\
\hline \multicolumn{6}{|c|}{ Resíduos de nabiça } \\
\hline Disco liso & $89 \mathrm{Ba}$ & $115 \mathrm{Aa}$ & $115 \mathrm{Ab}$ & $131 \mathrm{Ab}$ & $112 \mathrm{~B}$ \\
\hline Disco corrugado & $103 \mathrm{Aa}$ & $105 \mathrm{Aa}$ & $121 \mathrm{Aab}$ & $124 \mathrm{Ab}$ & $113 \mathrm{~B}$ \\
\hline Disco ondulado & $104 \mathrm{Ba}$ & $103 \mathrm{Ba}$ & $141 \mathrm{Aa}$ & $151 \mathrm{Aa}$ & $125 \mathrm{~A}$ \\
\hline Média & $98 \mathrm{~b}$ & $108 \mathrm{~b}$ & $126 \mathrm{a}$ & $135 a$ & \\
\hline \multicolumn{6}{|c|}{$\begin{array}{l}\text { DMS discos }=10,21 ; \text { DMS cargas }=12,99 \\
\text { DMS carga d. disco }=22,51 ; \text { DMS disco d. carga }=20,42 ; \\
C V=10,01 \%\end{array}$} \\
\hline
\end{tabular}

* Médias seguidas por letras distintas, maiúsculas nas linhas e minúsculas nas colunas, diferem pelo "Teste Tukey" a 0,05 de probabilidade

horizontal, com exceção nas culturas do milho e da aveia preta cujas diferenças se devem às profundidades de penetração do disco em que o disco liso sempre resulta em maior profundidade, ocasionando maior força horizontal.

A cobertura vegetal do sorgo foi aquela que apresentou os maiores valores de força horizontal $(167 \mathrm{~N})$ seguida do milho, aveia preta, triticale e nabiça, respectivamente, o que se deve à maior dificuldade de corte dos discos na palha do sorgo.

Observa-se também, com o aumento da profundidade de trabalho, que ocorreu aumento no requerimento de força horizontal, fato também observado por Silva \& Benez (2005) e Germino \& Benez (2006) uma vez que, quanto maior a profundidade de trabalho maior também a resistência oferecida pelo solo ao equipamento.
Os resultados concordam com os de Harrison (1977) que, conduzindo estudos de campo usando discos esféricos, concluiu que com o aumento na profundidade de corte do solo ocorreu um incremento de força horizontal da ordem de $42 \%$, força vertical de $31 \%$ e na força lateral de $29 \%$.

\section{Força vertical}

Os resultados médios de força vertical são apresentados na Tabela 4.

O mecanismo de corte do tipo disco liso obteve os maiores valores de força vertical em relação aos demais mecanismos para as coberturas de milho e sorgo. Isto pode ser explicado pela presença dos restos vegetais na superfície do solo com maior dificuldade de corte e pela função do disco em penetrar

Tabela 4. Valores médios* de força vertical (N) em diferentes coberturas vegetais

\begin{tabular}{|c|c|c|c|c|c|}
\hline \multirow{2}{*}{$\begin{array}{l}\text { Disco } \\
\text { de corte }\end{array}$} & \multicolumn{4}{|c|}{ Níveis de carga (N) } & \multirow{2}{*}{ Média } \\
\hline & 750 & 1500 & 2250 & 3000 & \\
\hline \multicolumn{6}{|c|}{ Resíduos da cultura do milho } \\
\hline Disco liso & $119 \mathrm{Ba}$ & $138 \mathrm{Aa}$ & $130 \mathrm{ABa}$ & 128 ABab & $129 \mathrm{~A}$ \\
\hline ugado & $89 \mathrm{Cb}$ & $114 \mathrm{ABb}$ & $103 \mathrm{BCb}$ & $118 \mathrm{Ab}$ & $106 \mathrm{~B}$ \\
\hline Disco 0 & $94 \mathrm{Bb}$ & $128 \mathrm{Aa}$ & $136 \mathrm{Aa}$ & $133 \mathrm{Aa}$ & $122 \mathrm{~A}$ \\
\hline Média & $101 \mathrm{~b}$ & $126 \mathrm{a}$ & 123 a & $126 a$ & \\
\hline \multicolumn{6}{|c|}{$\begin{array}{l}\text { DMS discos = 6,76; DMS cargas = } 8,61 ; \\
\text { DMS carga d. disco = 14,92; DMS disco d. carga = 13,53; } \\
\text { CV = } 12,54 \%\end{array}$} \\
\hline \multicolumn{6}{|c|}{ Resíduos da cultura do sorgo } \\
\hline Disco liso & $124 \mathrm{Ba}$ & $133 \mathrm{ABa}$ & $141 \mathrm{Aa}$ & $148 \mathrm{Ab}$ & $137 \mathrm{~A}$ \\
\hline ugado & $98 \mathrm{Cb}$ & $116 \mathrm{Bb}$ & 138 & $125 \mathrm{ABC}$ & $119 \mathrm{~B}$ \\
\hline Disco ondulado & $114 \mathrm{Ca}$ & $97 \mathrm{DC}$ & $146 \mathrm{Ba}$ & $167 \mathrm{Aa}$ & 1. \\
\hline Média & $112 \mathrm{~b}$ & $116 \mathrm{~b}$ & $142 \mathrm{a}$ & 146 a & \\
\hline \multicolumn{6}{|c|}{$\begin{array}{l}\text { DMS discos = 7,30; DMS cargas = 9,29; } \\
\text { DMS carga d. disco = 16,08; DMS disco d. carga = 14,59; } \\
\text { CV = 16,50\% }\end{array}$} \\
\hline \multicolumn{6}{|c|}{ Resíduos da cultura da aveia preta } \\
\hline Disco 1 & $103 \mathrm{Ba}$ & $99 \mathrm{Bb}$ & $131 \mathrm{Aa}$ & $120 \mathrm{ABa}$ & 113 \\
\hline ugado & $112 \mathrm{Aa}$ & 117 & 105 & $116 \mathrm{Aa}$ & 112 \\
\hline Disco ondulado & $98 \mathrm{Ba}$ & $123 \mathrm{ABa}$ & $128 \mathrm{Aa}$ & $121 \mathrm{ABa}$ & 117 \\
\hline Média & $104 \mathrm{~b}$ & $113 a b$ & $121 \mathrm{a}$ & $119 a b$ & \\
\hline \multicolumn{6}{|c|}{$\begin{array}{l}\text { DMS discos = 11,25; DMS cargas = 14,33; } \\
\text { DMS carga d. disco = 24,82; DMS disco d. carga = 22,51; } \\
C V=11,34 \%\end{array}$} \\
\hline \multicolumn{6}{|c|}{ Resíduos da cultura do triticale } \\
\hline Disco liso & $89 \mathrm{Ba}$ & $86 \mathrm{Bb}$ & $97 \mathrm{BC}$ & $115 \mathrm{Aa}$ & $97 \mathrm{C}$ \\
\hline Disco corrugado & $94 \mathrm{Ba}$ & $114 \mathrm{Aa}$ & 11 & $109 \mathrm{ABa}$ & 108 \\
\hline Disco ondulado & $103 \mathrm{Ca}$ & $127 \mathrm{ABa}$ & $137 \mathrm{Aa}$ & $118 \mathrm{BCa}$ & $122 \mathrm{~A}$ \\
\hline Média & $95 \mathrm{~b}$ & $109 a$ & $117 \mathrm{a}$ & $114 \mathrm{a}$ & \\
\hline $\begin{array}{l}\text { DMS discos }= \\
\text { DMS carga } d . d\end{array}$ & $\begin{array}{l}\text { DMS ca } \\
=18,44\end{array}$ & $\begin{array}{l}\text { gas }=10 \\
\text { DMS disc }\end{array}$ & d. c & 16,13 & \\
\hline
\end{tabular}

\begin{tabular}{|c|c|c|c|c|c|}
\hline \multicolumn{6}{|c|}{ Resíduos de nabiça } \\
\hline Disco liso & $101 \mathrm{Aa}$ & $89 \mathrm{Ab}$ & $110 \mathrm{Aab}$ & $112 \mathrm{Ab}$ & $103 \mathrm{~B}$ \\
\hline Disco corrugado & $102 \mathrm{ABa}$ & $110 \mathrm{ABa}$ & $97 \mathrm{Bb}$ & $122 \mathrm{Aab}$ & $108 \mathrm{~B}$ \\
\hline Disco ondulado & $92 \mathrm{Ba}$ & $121 \mathrm{Aa}$ & $127 \mathrm{Aa}$ & $136 \mathrm{Aa}$ & $119 \mathrm{~A}$ \\
\hline Média & $98 \mathrm{~b}$ & $106 \mathrm{~b}$ & $112 a b$ & $123 a$ & \\
\hline \multicolumn{6}{|c|}{$\begin{array}{l}\text { DMS discos = 10,43; DMS cargas = 13,38; } \\
\text { DMS carga d. disco = 23,01; DMS disco d. carga = 20,87; } \\
C V=10,92 \%\end{array}$} \\
\hline
\end{tabular}
pelo "Teste Tukey" a 0,05 de probabilidade 
no solo. Esses valores estão próximos aos encontrados por Mion \& Benez (2008). Entretanto, pode-se observar, para as coberturas de triticale e nabiça, que o disco de corte do tipo ondulado foi o que obteve os maiores valores de força vertical devido a resistência oferecida pelo solo à penetração e pela palha presente na superfície ser cortada de modo mais fácil; com isto, a força vertical requerida se encontra em função da resistência do solo e não da palha, concordando com Chang et al. (1983), Portella (1983) e Kushwaha et al. (1986).

\section{Força lateral}

Os resultados de força média lateral são apresentados na Tabela 5. Verifica-se que, independente da carga vertical aplicada, o disco ondulado apresentou valores de força lateral

Tabela 5. Valores médios* de força lateral (N) para as diferentes coberturas vegetais

\begin{tabular}{|c|c|c|c|c|c|}
\hline \multirow{2}{*}{$\begin{array}{l}\text { Disco } \\
\text { de corte }\end{array}$} & \multicolumn{4}{|c|}{ Níveis de carga (N) } & \multirow{2}{*}{ Média } \\
\hline & 750 & 1500 & 2250 & 3000 & \\
\hline \multicolumn{6}{|c|}{ Resíduos da cultura do milho } \\
\hline Disco liso & $135 \mathrm{Bb}$ & $174 \mathrm{Ab}$ & $148 \mathrm{Bb}$ & $127 \mathrm{BC}$ & $146 \mathrm{~B}$ \\
\hline Disco & 131 & 147 & & 159 & $9 \mathrm{~B}$ \\
\hline Disco o & $196 \mathrm{Ca}$ & $244 \mathrm{Ba}$ & $247 \mathrm{Ba}$ & $278 \mathrm{Aa}$ & $241 \mathrm{~A}$ \\
\hline Média & $154 \mathrm{~b}$ & $188 \mathrm{a}$ & $186 \mathrm{a}$ & $188 \mathrm{a}$ & \\
\hline \multicolumn{6}{|c|}{$\begin{array}{l}\text { DMS discos = 9,97; DMS cargas = 12,69; } \\
\text { DMS carga d. disco = 21,99; DMS disco d. carga = 19,95; } \\
\text { CV }=16,41 \%\end{array}$} \\
\hline \multicolumn{6}{|c|}{ Resíduos da cultura do sorgo } \\
\hline sco liso & $142 \mathrm{Bb}$ & $187 \mathrm{Aa}$ & $174 \mathrm{Ab}$ & $177 \mathrm{Ab}$ & $170 \mathrm{~B}$ \\
\hline Igado & $136 \mathrm{ABb}$ & $163 \mathrm{~A}$ & $128 \mathrm{BC}$ & $139 \mathrm{ABC}$ & $142 \mathrm{C}$ \\
\hline lulado & $204 \mathrm{Ba}$ & $188 \mathrm{Ba}$ & $211 \mathrm{Ba}$ & $283 \mathrm{Aa}$ & $222 \mathrm{~A}$ \\
\hline Média & $161 \mathrm{c}$ & $179 \mathrm{~b}$ & 171 bc & 199 a & \\
\hline \multicolumn{6}{|c|}{$\begin{array}{l}\text { DMS discos = 14,54; DMS cargas = 18,51; } \\
\text { DMS carga d. disco = 32,06; DMS disco d. carga = 29,07; } \\
\text { CV }=9,41 \%\end{array}$} \\
\hline \multicolumn{6}{|c|}{ Resíduos da cultura da aveia preta } \\
\hline isco liso & $95 \mathrm{Bb}$ & $125 \mathrm{Aa}$ & $121 \mathrm{Ab}$ & $120 \mathrm{Ac}$ & $115 \mathrm{~B}$ \\
\hline sco corrugado & $108 \mathrm{Bab}$ & 103 & 138 & $144 \mathrm{Ab}$ & $123 \mathrm{~B}$ \\
\hline dulado & $129 \mathrm{Ba}$ & 106 & $169 \mathrm{Aa}$ & $193 \mathrm{Aa}$ & $149 \mathrm{~A}$ \\
\hline Média & $110 \mathrm{~b}$ & $111 \mathrm{~b}$ & $142 \mathrm{a}$ & $152 \mathrm{a}$ & \\
\hline \multicolumn{6}{|c|}{$\begin{array}{l}\text { DMS discos = 11,03; DMS cargas = 14,04; } \\
\text { DMS carga d. disco = 24,32; DMS disco d. carga = 22,05; } \\
\text { CV }=12,70 \%\end{array}$} \\
\hline \multicolumn{6}{|c|}{ Resíduos da cultura do triticale } \\
\hline Disco & 108 & 93 & 118 & 107 & $107 \mathrm{C}$ \\
\hline ugado & 101 & 115 & 13 & 119 & $117 \mathrm{~B}$ \\
\hline Disco ondulado & 127 & 122 & 147 & 130 & 101 \\
\hline Média & $112 \mathrm{~b}$ & $110 \mathrm{~b}$ & $134 \mathrm{a}$ & $119 \mathrm{~b}$ & \\
\hline \multicolumn{6}{|c|}{$\begin{array}{l}\text { DMS discos = 9,34; DMS cargas = 11,89; } \\
\text { DMS carga d. disco = ns; DMS disco d. carga = ns; } \\
\text { CV }=9,07 \%\end{array}$} \\
\hline \multicolumn{6}{|c|}{ Resíduos de nabiça } \\
\hline Disco liso & $87 \mathrm{Bb}$ & $93 \mathrm{Bb}$ & 92 & $134 \mathrm{Aa}$ & $101 \mathrm{~B}$ \\
\hline Disco corrugado & 103 Aab & $104 \mathrm{Ab}$ & $107 \mathrm{Aab}$ & $106 \mathrm{Ab}$ & $105 \mathrm{~B}$ \\
\hline Disco ondulado & $118 \mathrm{Ba}$ & $129 \mathrm{Ba}$ & $119 \mathrm{Ba}$ & $156 \mathrm{Aa}$ & $130 \mathrm{~A}$ \\
\hline Média & $103 \mathrm{~b}$ & $109 \mathrm{~b}$ & $106 \mathrm{~b}$ & $132 \mathrm{a}$ & \\
\hline \multicolumn{6}{|c|}{$\begin{array}{l}\text { DMS discos = 11,98; DMS cargas = 15,25; } \\
\text { DMS carga d. disco = 26,42; DMS disco d. carga = 23,96; } \\
C V=12,28 \%\end{array}$} \\
\hline
\end{tabular}
pelo "Teste Tukey" a 0,05 de probabilidade superiores aos dos demais discos de corte, sinalizando tendência do equipamento possuir força lateral maior para o lado em que se dá a declividade do terreno, o que pode causar problemas de avarias nos discos de corte quando as semeadoras-adubadoras estiverem trabalhando em local com alta declividade, como no caso desses experimentos. Os resultados de força lateral também podem ter ocorrido pela interação solo-metal causando fricção e gerando forças laterais aumentando, desta forma, a resistência ao rolamento. Tais resultados podem ser diferentes quando se trabalha em condições de baixa declividade. Salienta-se que esses valores de força lateral para os diferentes tipos de discos de corte são superiores aos encontrados por Morrison Júnior et al. (1996) e Mion \& Benez (2008) passível de ser explicado em razão da maior declividade do terreno.

Para o mecanismo de corte do tipo disco ondulado o aumento da carga vertical proporcionou maiores valores de força lateral mostrando que este comportamento se deve, sem dúvida, ao desenho da ferramenta. A força lateral para os discos do tipo corrugado e liso mostrou comportamento semelhante; outro fator importante a ressaltar é que esses valores obtidos podem ser devidos à variação de direção do deslocamento do trator, ou seja, o trator nunca trabalha em linha reta, o que resulta em movimentação lateral e esforço diferenciado dos discos.

\section{CONCLusões}

1. Quanto maior a quantidade de massa seca maior também é a demanda das forças horizontal e vertical.

2. Quanto maior a carga vertical aplicada maior também a área de solo mobilizado.

3. A semeadora deve ser regulada com a carga mínima para que haja o corte adequado da palha.

\section{LITERATURA CITADA}

ASAE - American Society of Agricultural Engineers. Agricultural machinery management data. In: American Society of Agricultural Engineers. ASAE standards 1999. Standards engineering practices data. Saint Joseph: ASAE, 1999. p.359-366.

Aratani, R. G.; Maria, I. C. de; Castro, O. M. de; Peche Filho, A.; Duarte, A. P.; Kanthack, R. A. D. Desempenho de semeadorasadubadoras de soja em Latossolo Vermelho muito argiloso com palha intacta de milho. Revista Brasileira de Engenharia Agrícola eAmbiental, v.10, p.517-522, 2006.

Beutler, A. N.; Centurion, J. F.; Silva, A. P. da. Soil resistance to penetration and least limiting water range for soybean yield in a haplustox from Brazil. Brazilian Archives of Biologyand Technology, v.48, p.863-871, 2005.

Chang, C. S.; Portella, J. A.; Kumagai, I. Exigência da força nos sistemas que utilizam tração para o plantio direto. In: Congresso Brasileiro de Engenharia Agrícola, 13, 1983, Rio de Janeiro. Anais... Rio de Janeiro: Sociedade Brasileira de Engenharia Agrícola,1983. p.130-134. 
Coelho, H.; Fernandes, H. C.; Campos, D. S.; Teixeira, M. M.; Leite, D. M. Deslizamento de rodados de tratores de pneus em diferentes operações agrícolas. Revista Ceres, v.59, p.330-336, 2012.

Gabriel Filho, A.; Lanças, K. P.; Leite, F.; Acosta, J. J. B.; Jesuíno, P. R. Desempenho de trator agrícola em três superfícies de solo e quatro velocidades de deslocamento. Revista Brasileira de Engenharia Agrícola e Ambiental, v.14, p.333339, 2010.

Germino, R.; Benez, S. H. Ensaio comparativo em dois modelos de hastes sulcadoras para semeadoras-adubadoras de plantio direto. Energia na Agricultura, v.21, p.85-92, 2006.

Harrison, H. P. Soil reaction forces for disks from field measurements. Transaction of American Society of Agricultural Engineers, v.20, p.836-838, 1977.

Llanillo, R. F.; Richart, A.; Tavares Filho, J.; Guimarães, M. F.; Ferreira, R. R. M. Evolução de propriedades físicas do solo em função dos sistemas de manejo em culturas anuais. Semina: Ciências Agrárias, v 27, p.205-220, 2006.

Kushwaha, R. L.; Vaishanav, A. S.; Zoerb, G. C. Soil bin evaluation of disccoulters under no-till crop residue conditions. Transaction of American Society of Agricultural Engineers, v.29, p.40-44, 1986.

Mahl, D. Desempenho de semeadoras-adubadoras de milho (Zea mays L.) em sistema de plantio direto. Botucatu: FCA/ UNESP, 2002. 160p. Dissertação Mestrado

Mello, L. M. M.; Pinto, E. R.; Yano, É. H. Distribuição de sementes e produtividade de grãos da cultura do milho em função da velocidade de semeadura e tipos de dosadores. Engenharia Agrícola, v.23, p.563-567, 2003.
Mion, R. L.; Benez, S. H. Esforços em ferramentas rompedoras de solo de semeadoras de plantio direto. Ciência e Agrotecnologia, v.32, p.1594-1600, 2008.

Morrison Júnior, J. E.; Hendrick III, J. G.; Schafer, R. L. Soil forces on coulter and disc-opener combinations. Transaction of American Society of Agricultural Engineers, v.39, p.369-376, 1996.

Portella, J. A. Um estudo preliminar de forças atuantes de elementos rompedores de semeadoras comerciais. Campinas: UNICAMP, 1983. 69p. Dissertação Mestrado

Santos, A. P.; Volpato, C. E. S.; Tourino, M. C. C. Desempenho de três semeadoras-adubadorasde plantio direto paraa cultura do milho. Ciência e Agrotecnologia, v.32, p.540-546, 2008.

Schuler, R. T.; Wood, R. K. Soil compaction.In: Conservation tillage systems and management: Crop residue management with no-till, ridge-till, mulch-till. Ames: Mid west Plan Service, 1992. p.42-45.

Silva, V. R.; Reinert, D. J.; Reichert, J. M. Densidade do solo, atributos químicos e sistema radicular do milho afetados pelo pastejo e manejo do solo. Revista Brasileira de Ciência do Solo, v.24, p.191-199, 2000.

Silva, P. R. A.; Benez, S. H. Avaliação de forças resultantes de mecanismos sulcadores de semeadoras-adubadoras de plantio direto. Energia na Agricultura, v.20, p.76-82, 2005.

Siqueira, R.; Casão Júnior, R. Trabalhador no cultivo de grãos e oleaginosas: Máquinas para manejo de coberturas e semeadura no sistema de plantio direto. Curitiba: SENARPR. 2004. 88p. 